\title{
La relevancia moral de la identidad cultural ${ }^{*}$
}

\section{Luis Villavicencio Miranda**}

\begin{abstract}
Resumen: Este artículo presenta argumentos a favor del diseño de políticas públicas sensibles al desafío multicultural y, en especial, a la circunstancia de que la cultura es un elemento crucial para la identidad individual. En primer lugar, repasa la querella entre liberales y comunitaristas como la fuente del actual debate. Luego, analiza la reacción de Rawls ante la crítica comunitarista. En tercer lugar, expone críticamente las tres grandes concepciones-modelo que intentan hacerse cargo del pluralismo cultural. Y, para finalizar, esboza una posible respuesta a las demandas de las minorías étnicas.
\end{abstract} cultural.

Palabras clave: liberalismo, comunitarismo, multiculturalismo, identidad

\section{The moral relevance of cultural identity}

Abstract: This article presents arguments in favor of designing public policies sensitive to multicultural challenge, especially taking into account that culture is a crucial element for individual identity. First, it reviews the dispute between liberals and communitarians as the source of the current debate. Second, it analyzes the Rawls's response to communitarian critique. Third, it exposes critically the three most important model-conceptions that pronounce about cultural pluralism. And finally, it outlines a possible response to the demands of ethnic minorities. identity.

Key words: liberalism, communitarianism, multiculturalism, cultural

\section{A relevância moral da identidade cultural}

Resumo: O presente artigo apresenta argumentos para a formulação de políticas públicas sensíveis ao desafio multicultural e, em especial, ao fato de que a cultura é um elemento crucial para a identidade individual. Em primeiro lugar, analisa a disputa entre liberais e comunitaristas como fonte do debate atual sobre o tema. Em seguida, analisa a reação de Rawls em face da crítica comunitarista. Em terceiro lugar, expõe criticamente as três grandes concepções-modelo que pretendem dar conta do pluralismo cultural. E, para finalizar, esboça uma resposta possível para as demandas das minorias étnicas. cultural.

Palavras-chave: liberalismo, comunitarismo, multiculturalismo, identidade

\footnotetext{
${ }^{*}$ Este artículo forma parte del proyecto de investigación $\mathrm{N}^{\circ} 11080006$ financiado por FONDECYT, titulado "Identidad y autonomía en sociedades multiculturales. Bases para una propuesta conciliatoria”.

** Universidad de Valparaíso, Valparaíso, Chile. E-mail: luis.villavicencio@uv.cl
} 


\section{Introducción}

Hasta la década de los '80 del siglo pasado, la discusión sobre la justicia estuvo marcada, predominantemente, por la posibilidad de argumentar a favor de una forma de liberalismo igualitario -representada paradigmáticamente por la justicia como equidad de Rawls (1971)- que suscribía una concepción individualizada y no agregativa de los derechos individuales bajo el paradigma de la distribución equitativa de esos mismos derechos (Beltrán 2002: 371-374; Gargarella 1999). Hoy esa aproximación se ha visto al menos parcialmente desplazada desde la entrada en escena de los comunitaristas y, particularmente, desde la constatación que ese paradigma de la distribución debe operar al interior de Estados que son, casi siempre, pluralistas desde el punto de vista de las culturas que convergen en su seno.

En las líneas que siguen intentaré exponer, partiendo de esa constatación indiscutible, argumentos teóricos a favor del diseño de políticas públicas sensibles al desafío multicultural y, en particular, al hecho de que la cultura es un elemento crucial en la conformación de la identidad individual. Para ello, en primero lugar, repasaré la querella entre liberales y comunitaristas como la fuente de la cual se nutre el actual debate, lo que me permitirá clarificar en qué sentido -y en cuál no- la crítica comunitarista da en el blanco. En segundo lugar, analizaré cómo reacciona Rawls ante la crítica comunitarista y expondré por qué dicha respuesta es insuficiente. En tercer lugar, me concentraré en el modo en que deberíamos actuar frente al desafío multicultural como expresión más relevante de aquello que el liberalismo deja sin una respuesta satisfactoria, presentando críticamente las tres grandes concepciones-modelo que intentan hacerse cargo del pluralismo cultural. Y, para terminar, esbozaré qué camino deberíamos recorrer para dar una respuesta adecuada a las demandas de las minorías étnicas asumiendo como presupuesto que los Estados no admitirán la fragmentación política.

\section{Liberalismo ortodoxo versus comunitarismo}

La disputa entre liberales y comunitaristas ha sido una de las grandes polémicas que ha arrinconado en dos trincheras, aparentemente irreconciliables, a los filósofos morales y políticos. Este debate enfrenta a dos posiciones teóricas extremas -entre una esperable multiplicidad de posiciones intermedias- y a dos modos distintos de comprender y organizar la justicia de las instituciones sociales básicas. Así, los liberales, por un lado, postulan la posibilidad de justificar un catálogo de derechos humanos que encuentra su fundamento último en la idea de autonomía individual, o sea, 
en la posibilidad que tiene cada agente moral de enfrentarse por sí mismo a las opciones más básicas de su vida y conducirla del modo más consecuente con sus convicciones; $y$, los comunitaristas, por otro lado, sostienen que el ser humano en general, y en particular el ciudadano de las sociedades complejas, no puede ser entendido al margen de las vinculaciones sociales que lo constituyen como sujeto, imposibilitando que la validez de cualquier moralidad y de toda política se emancipe de los horizontes de significado que sitúan a cada persona no como un ente autónomo anterior a los valores y fines sociales sino más bien como un ente definido, precisamente, por aquellos horizontes.

Para el liberalismo, particularmente en la versión de Rawls (1971, 1996, y 2002), más allá de cualquier pertenencia a un grupo o comunidad, los individuos tienen la capacidad de cuestionar las relaciones con ese grupo o comunidad hasta el punto incluso de separarse o renunciar totalmente a ellas. Para el comunitarismo, por el contrario, nuestra identidad como personas se encuentra profundamente marcada por nuestra pertenencia a ciertos grupos en los cuales estamos insertos y de los que si alguna vez fuéramos aislados dejaríamos de ser, sencillamente, lo que realmente somos, esto es, perderíamos el único modo por medio del cual podemos ser auténticos agentes morales. En este escenario, para el liberalismo, el pluralismo moral, político, religioso y cultural es una característica estructural de las sociedades modernas y nada de desastroso hay en ello, sino que es el resultado obvio de las actividades de la razón humana en contextos institucionales perdurablemente libres (Rawls: 1996). Pero, para las tesis comunitaristas, este pluralismo es un síntoma claro de un proceso de descomposición moral, que se traduce en la particularización de la comunidad en individuos atomizados que han perdido toda consistencia e integración cultural (Taylor: 1990, 1994, 1996, y 1997; MacIntyre: 1987, 1992, 1994, y 2006; Sandel: 1996, 1998, 2000, y 2006; Walzer: 1993, 1996, 1998, y 2006).

Dentro de esta aproximación general, hay algunos aspectos de la arremetida comunitarista que resultan particularmente relevantes para los efectos de esta exposición:

a) En primer lugar, la crítica a la concepción de persona en que se sustenta el liberalismo -especialmente el rawlsiano (Véase Rawls 1971, 1986, 1996, 2001a, 2001b, y 2002)-. Según ésta, particularmente en la versión de Sandel (2000: 31-89), la identidad del agente moral no puede disociarse -a costa de caer en una concepción falsa- de que todos nos concebimos como miembros activos de grupos comunitarios en los cuales estamos insertos, los cuales nos dotan de las herramientas indispensables para que podamos responder a la pregunta de quiénes somos y, además, alcanzar ciertos bienes imprescindibles que sólo adquieren sentido en un contexto mayor que el del individuo considerado aisladamente.

b) En segundo lugar, la objeción que niega la posición ontológica sobre la cual se fundaría el liberalismo, esto es, el atomismo o individualis- 
mo asocial (Véase principalmente Taylor 1990: 107-123; 1996: 36-38; 1997 : 335-372; y MacIntyre: 1987, y 1994). Para el comunitarismo, toda concepción racional de la identidad del agente moral exige que reconozcamos la trascendencia ineludible de los marcos referenciales y las distinciones cualitativas. Pero estos marcos son indefectiblemente sociales y, en consecuencia, sólo pueden ser adquiridos, adecuadamente comprendidos y protegidos en razón de estrechos vínculos constitutivos entre el individuo y la comunidad, por lo que cualquier teoría moral que plantee la prioridad del individuo sobre la sociedad que lo cobija, y la prioridad paralela de lo justo sobre lo bueno, es conceptualmente contradictoria. Más aún, los comunitaristas postulan que dicho atomismo acarrearía consecuencias indeseables para las propias tesis normativas que defiende el liberalismo, por un lado, descuidar las fuentes comunitarias de los bienes que constituyen y configuran esos valores y, por otro, darle un soporte inestable o derechamente contradictorio a las demandas igualitaristas que deben imponerse a los sujetos considerados aisladamente (Sandel 2000: 107 y 108).

c) En tercer y último lugar, la crítica que sostiene la falsedad de la prioridad epistemológica de la justicia sobre lo bueno y, por implicación, la imposibilidad de la neutralidad liberal (Sandel 2000: 186-216; MacIntyre 1994: 313-330). En vez de esa pretendida primacía cualquier teoría ética coherente debería asociarse a una cierta idea del bien y de los fines compartidos por una determinada comunidad. Para poder justificar la tesis de la prioridad de lo justo el liberalismo tiene que echar mano no a una concepción débil del bien sino a una de carácter muy robusto que se sustentaría en una serie de distinciones cualitativas unida a una particular concepción de la naturaleza humana.

Frente a los reproches apuntados, una lectura filosóficamente atenta de los presupuestos liberales y, en particular, de la versión más sofisticada y vigorosa de esa tradición de pensamiento, esto es, la justicia como equidad de Rawls tal como fue presentada en A Theory of Justice (1971) más las aclaraciones y correcciones integradas a la teoría por el propio filósofo estadounidense -particularmente en su trabajo "El constructivismo kantiano en la teoría moral” (1986: 209-262)- permite enfrentar con éxito dos aspectos parciales de las críticas comunitaristas resumidas más arriba: por una parte, aquellas que acusaban a la justicia como equidad de "ocultar" una cierta concepción de la persona sin la cual la teoría se encontraba huérfana de una adecuada fundamentación que fortaleciera el precario argumento contractual en el que, al menos explícitamente, parecía descansar toda la construcción rawlsiana; y, por otra, las objeciones que atribuían a la justicia como equidad una excesiva abstracción y universalidad que la exponía a los típicos reproches que pueden hacérsele a una doctrina de inspiración kantiana (Peña 2002). Así pues, apoyándose en el constructivismo kantiano, Rawls sostiene que la teoría se sustenta efectivamente en una concepción de la persona que al ser normativa no la compromete con ninguna doctrina o antropología metafísica sobre la naturaleza humana y, al mismo tiempo, le permite obviar la crítica de caer en la abstracción y de tener pretensiones universalistas puesto que la justicia como equidad se 
funda en una noción concreta de los agentes morales, que hunde sus raíces en ciertas intuiciones compartidas propias de la cultura política democrática moderna (Rawls 1986: 209-262). Además de lo anterior, el filósofo estadounidense puede defenderse de la crítica de que la teoría rawlsiana sería emotivista o subjetivista en el sentido de que el acuerdo al que llegan las partes en la posición original es un tipo de elección radical, es decir, un tipo de elección que no se basa en razones, defendiendo la razonabilidad como una forma de objetividad propia de aquellos que comparten la concepción de persona que inspira el mecanismo de representación de la situación ideal de deliberación (Rawls: 1986, 192-208). Por último, frente a la acusación de ser una teoría atomista, la justicia como equidad puede replicar que los intereses de los individuos dependen de las instituciones existentes y de los principios de justicia que las gobiernan por lo que, no obstante la base metodológica individualista del liberalismo, la teoría de la justicia da cabida suficiente a la naturaleza social de los individuos (Rawls 2001b: 383-389).

\section{La réplica liberal. El liberalismo político y la corrección interna de la justicia como equidad}

A pesar de la fortaleza de la defensa rawlsiana recién expuesta, una porción relevante de la crítica comunitarista mantiene su fuerza al derivarse de ella que la justicia como equidad se funda en una concepción particular de la autonomía que, aunque no sea metafísica como señalan los comunitaristas, sí reposa en una doctrina comprehensiva que compromete la neutralidad de la teoría (Nino 1996: 123-135). Rawls admite en El liberalismo político (1996) que la justicia como equidad en su primera versión padecía dos graves problemas internos: primero, presentaba a la concepción de justicia como parte de una concepción más general que Rawls denomina corrección como equidad; y, segundo, presuponía que en una sociedad bien ordenada, de conformidad con los principios de justicia, los ciudadanos aceptarían sus principios en tanto se encuentran identificados con una visión comprehensiva de raíz kantiana (Rawls 1996: 40 y 41). De este modo, este grave problema acarrea que la justicia como equidad tal como es construida en A Theory of Justice -específicamente en la tercera parte de la obra- exige demasiado a los individuos para poder alcanzar la estabilidad, o sea, demanda que los ciudadanos converjan en torno a una misma doctrina comprehensiva, lo que resulta incompatible con la rica e irreductible pluralidad que caracteriza a las sociedades democráticas modernas.

La estrategia desarrollada por el filósofo estadounidense para salvar esta deficiencia consiste fundamentalmente en replantear la justicia como equidad como una concepción estrictamente política, es decir, que su justificación descansa en valores políticos y, por ende, no debe ser considerada como parte de una doctrina moral, religiosa o filosófica más completa o comprehensiva. ¿Cumple Rawls su objetivo? Los autores liberales, luego de las críticas comunitaristas, han optado por replantear sus tesis en cuatro 
direcciones opuestas: unos, han buscado refundar la neutralidad liberal, como es el caso del segundo Rawls de El liberalismo político; otros, han redefinido el liberalismo aceptando cierta conexión entre el bienestar de los individuos y el de la comunidad política a la cual pertenecen, como por ejemplo Dworkin (1993, 1996a, 1996b, y 2000), configurando una especie de liberalismo que podría denominarse comprehensivo o integrado; algunos han postulado la posibilidad de defender los valores liberales sin la necesidad de recurrir a ningún tipo de justificación, como sería el caso del liberalismo sin fundamentos que defiende Rorty (1991 y 1996); y, todavía, ciertos autores han desarrollado un liberalismo comprehensivo -al igual que el dworkiniano- pero perfeccionista, como por ejemplo la moralidad de la libertad que sostiene Raz (1986 y 2001). Aquí sólo me limitaré a esbozar si la estrategia desarrollada por el segundo Rawls le permite esquivar con éxito las críticas comunitaristas, lo que me permitirá además conectar este debate con los desafíos que desde el pluralismo étnico pueden hacérsele a la concepción liberal de la ciudadanía.

Mi hipótesis es que dos aspectos de la construcción liberal deben ser readecuados, por un lado, la filosofía moral -en este caso la que informa el punto de vista liberal- debe tener contacto con la práctica moral que se alimenta de circunstancias históricas determinadas; y, por otro, la neutralidad liberal, fundamentada en el valor de la autonomía, requiere algún tipo de sustento en una concepción particular del bien. Así pues, esta forma de acercarse a la teoría liberal defiende que no hay una conexión necesaria entre las posturas metaéticas del liberalismo clásico (la universalidad y la neutralidad) y las conclusiones normativas sustantivas que suscribe esa misma forma de liberalismo y que continuaría defendiendo uno corregido, atento a los reproches comunitaristas. Creo que las tesis sustantivas liberales pueden ser mantenidas modificando su soporte metaético y, lo que es decisivo, sin alterar trascendentalmente las consecuencias prácticas de la pretensión de universalidad de las tesis normativas liberales. O sea, la autonomía moral (quizá el valor más preciado del liberalismo) podría instalarse, manteniendo presupuestos metaéticos comunitaristas, allí donde aparentemente los significados históricos compartidos no la recibirían bien (Villavicencio 2007 y 2009).

El propio Rawls introdujo importantes modificaciones a la justicia como equidad en su obra El liberalismo político, alteraciones que buscan subsanar los defectos que A Theory of Justice presentaba por estar comprometida con una concepción comprehensiva y que implican, por ende, intentar subsanar los defectos apuntados en relación con la neutralidad de la teoría. De esta forma, esta reformulación puede ser vista como una respuesta a las críticas comunitaristas, especialmente las de Sandel, aunque no suponen la aceptación de que la justicia como equidad reposaba en una concepción metafísica errada de la persona (Rawls 1996: 57 y 58).

La apuesta de Rawls en El liberalismo político se sustenta en la apelación metodológica al constructivismo político que discurre en derredor de tres ejes: una concepción política de la persona, la idea de razón 
pública como criterio de justificabilidad, y la idea del consenso por superposición como explicación de la estabilidad (Mulhall y Swift 1996: 229255). El proceso de construcción procura modelar, al igual que en A Theory of Justice, una concepción normativa de persona -por oposición a una metafísica- pero que sólo es relevante respecto de aquellas cuestiones vinculadas a la estructura básica de la sociedad y expuesta, ya no como parte de una doctrina comprehensiva, sino como implícita en la cultura política pública. De esta forma, la adhesión a los principios de justicia sería posible aun cuando se profesaran distintas doctrinas comprehensivas sea que, por un lado, otorguen o no importancia preponderante a la participación en la vida política y, por otro, consideren o no constitutivos a los fines o vínculos privados. Además, el carácter restrictivo de la concepción política de persona evitaría comprometerse con la idea disputada de la racionalidad que entiende los juicios morales como meras preferencias.

De igual modo, la estabilidad ya no depende de la aceptación por parte de los ciudadanos y ciudadanas de una visión comprehensiva que incluya a la justicia como equidad, sino que se muestra como la concepción de la justicia que puede constituirse en el foco de un consenso por superposición de doctrinas comprehensivas inconmensurables entre sí, pero razonables. En fin, la razón pública se erige como el criterio que permite evaluar la justicia de las políticas llevadas adelante por el Estado, conforme a razones que no radiquen en el carácter superior de una determinada forma de vida, sino en argumentos que puedan ser aceptados por sujetos que mantienen profundas divergencias precisamente en ese sentido.

Con todo, el intento desplegado por Rawls para eludir cualquier compromiso con alguna doctrina comprehensiva se ve malogrado. Si bien el filósofo logra sortear con éxito las críticas que pretenden endosarle la adopción de una concepción política sustentada en razones estrictamente prudenciales, como si el liberalismo político fuera un mero modus vivendi (Rorty 1991 y 1996); y también aquellas objeciones que acusan al liberalismo político de comprometerse, a nivel político, con un escepticismo ético incompatible con la neutralidad que la teoría busca defender, no consigue, sin embargo, zafarse del reproche que culpa a la concepción política de caer en las redes de la paradoja de la razón pública o la sinrazón de la discontinuidad entre lo público y lo privado, que no tiene respuesta a la pregunta de por qué razones estaríamos dispuestos a dejar de lado nuestra propia doctrina comprehensiva cuando se discuten en el ámbito público los temas más fundamentales.

El modo en que Rawls resuelve el problema de la verdad y la objetividad en el dominio político lo lleva a sostener que toda doctrina comprehensiva, prima facie, tendría el derecho a intentar presentar en la arena política sus argumentos, pero al hacerlo debiera respetar las exigencias de lo razonable (Rawls 2002: 241-245). Estos requisitos mandan -como sucede en el caso de la doctrina que postula "que fuera de la iglesia no hay salvación”- descartar como una visión irrazonable cualquier idea comprehensiva que pretenda, mediante el uso coercitivo del poder político 
(un poder sobre el cual todos los ciudadanos y ciudadanas tienen una participación igual), imponer una concepción que afecta a algunas de las esencias constitucionales sobre las que, sin duda, las personas razonables, dadas las cargas del juicio, diferirán necesariamente. Esta postura por supuesto no demanda alegar la falsedad de la doctrina extra ecclesia nulla salus, sólo exige que comprendamos que es irrazonable usar el poder político para imponer nuestra propia visión religiosa, filosófica o moral completa en el ámbito público, aunque al mismo tiempo, como nos reclama la coherencia de la visión comprehensiva que profesamos, la estimemos verdadera.

Pues bien, la cuestión clave, creo, es que la respuesta rawlsiana al problema de la verdad u objetividad no es problemática por el método de elusión cuasiepistémico en que se funda (Cfr. Raz 2001: 74-79), sino en la dificultad de distinguir clara y establemente el ámbito público y el no público. El gran cuestionamiento que subyace a esta objeción es que la exclusión de las consideraciones morales sustentadas en las diferentes doctrinas comprehensivas, cuando se discuten cuestiones que tienen que ver con el diseño de la estructura básica de la sociedad, supone necesariamente tomar partido, en parte al menos, por la visión comprehensiva que se practica, es decir, implica suscribir una doctrina comprehensiva de corte liberal que defiende la separación entre lo político y no político.

Pero, ¿qué sucede cuando esa doctrina comprehensiva se sustenta en una cosmovisión cultural que choca con el ideario liberal? Considérense los siguientes ejemplos: Según Walzer (1993: 22), un bien como el pan, tan paradigmáticamente identificado como alimento básico, puede ser considerado por ciertas culturas como sagrado, ¿cómo podríamos comprender, sin escudriñar los horizontes comunes de una comunidad, el hecho de que una persona escoja morir de hambre que comer el pan que sólo está concebido en esa cultura para ser ofrecido a Dios? De la misma manera, supongamos que una persona se lava los pies en barro caliente todas las mañanas al amanecer produciéndose dolorosas lesiones en los pies (Taylor 1994: 72), ¿podríamos entender esa acción descontextualizada de la creencia compartida de que lavarse los pies en agua caliente todos los días es el acto litúrgico central de la religión que profesa voluntariamente esa persona? Obviamente, ninguno de estos ejemplos es decisivo para poner en jaque la distribución universal de la autonomía -el valor normativo más preciado para el liberalismo- porque precisamente presuponen decisiones autónomas, pero permite comprender que los marcos comunes de referencia, anclados necesariamente en doctrinas comprehensivas, no pueden ser irrelevantes, como pretende Rawls, para definir y comprender los derechos y principios liberales sustantivos. A un liberal, por lo tanto, no le quedará más opción que defender el valor de la autonomía no como una exigencia abstracta de la razón práctica, sino como la expresión de ciertos horizontes de sentido común compartidos entre todos aquellos que formamos parte de la cultura democrática moderna. De este modo, podrá considerar ilegítima una práctica social que, fundada en una religión impuesta coercitivamente, niega la autonomía plena a las mujeres y, por ello, sostener por las razones metaéticas correctas para su postura -que no son para nada universalistas- 
que a la mujer se le debe reconocer aquella noción de autonomía compartida, puesto que no existe ninguna razón dentro del marco común para negársela, salvo la más pura arbitrariedad que no es razón para nadie.

Veamos, ahora, una posibilidad más compleja. Un liberal, si no puede dejar a un lado los presupuestos metaéticos de la doctrina comprehensiva que profesa, sólo podría defender la libertad religiosa en aquellas culturas en las que tiene sentido hablar de un derecho a la libertad religiosa, o de cualquier derecho, puesto que son potestades que aseguran el ejercicio precisamente de la autonomía. Por lo tanto, si en una sociedad determinada no se comparte esta concepción de las personas morales como libres e iguales, hablar de derechos implicará un sin sentido, ya que el significado intersubjetivo que el término "derecho" expresa se encontrará fuera del vocabulario disponible para esa sociedad. Pues bien, aunque podamos poner en duda la veracidad de la circunstancia de que en algunas comunidades las personas nieguen el valor de la autonomía y de los derechos como un marco compartido, o creamos poco factible que voluntariamente alguien pueda someter su libertad a una religión integrista, o incluso más, dudemos fuertemente que en una sociedad donde no se reconozca la autonomía pueda darse la posibilidad de una opción en cualquier sentido plausible; la única conclusión razonable a la que podríamos llegar es que debemos ser capaces de extraer de esa cultura extraña los elementos necesarios para que sus propios miembros tengan la posibilidad de hacer inteligible y comprender el valor de la autonomía. Así, por ejemplo, la ablación genital que sufren millones de mujeres fundadas en motivos religiosos y también culturales sociales (Facchi 1999: 143-167), sólo podríamos potencialmente impedirla fomentando que dichas mujeres accedan a toda la información relevante para recrear las condiciones en que debiera darse una elección autónoma y confiar en que ninguna mujer, en esa posición, elegiría voluntariamente que le mutilaran el clítoris (Cfr. Comanducci 1996: 13-26).

Sin embargo, queda pendiente todavía la situación más extrema: ¿qué ocurre con las doctrinas comprehensivas o culturales que se empeñan en ser, desde un punto rawlsiano, irrazonables y despreciar por ende el valor de la autonomía? Volviendo al ejemplo, ¿cuál es la actitud moralmente correcta frente a una cultura que impone a las mujeres -contra su voluntad- la práctica de la ablación? Es precisamente en este punto donde se cuela sin más remedio el carácter comprehensivo del liberalismo político al excluir del consenso por superposición a aquellas doctrinas que están dispuestas a sacrificar la estabilidad y los principios de justicia en razón de la verdad interna de sus cosmovisiones. La pregunta que Rawls deja sin respuesta es por qué una doctrina comprehensiva no razonable en términos rawlsianos aceptaría la separación entre el ámbito público y no público si para ella, conforme a su visión comprehensiva, no tiene sentido alguno tratar a todos como ciudadanos libres e iguales que buscan los mejores principios para el trato recíproco si no se reconocen bajo ningún respecto como libres e iguales. $\mathrm{O}$, lo que viene a ser lo mismo, ¿por qué deberíamos tratarnos como semejantes si no nos vemos, desde nuestras culturas e identidades particulares, en ningún caso como semejantes? (Seleme 2004: 244 y 245). 


\section{Pluralismo y minorías culturales en sociedades complejas. Tres modelos para desarmar: el liberalismo igualitario, el culturalismo liberal y los multiculturalismos}

Desde muy antiguo han convivido, de muchas formas, diferentes culturas. Evidentemente el hecho del pluralismo cultural no es una novedad histórica, pero tres grandes factores -que distinguen las sociedades multiculturales contemporáneas de sus predecesoras- hacen indispensable la construcción de nuevas respuestas teóricas para encarar este fenómeno de antaño con renovadas categorías normativas. En primer lugar, la expansión de las ideas democráticas permite a las comunidades minoritarias resistirse a aceptar su estatus inferior y demandar no sólo iguales derechos, sino también una igual oportunidad de participar en la vida colectiva. En segundo lugar, el proceso de globalización económica y cultural deviene en inviable cualquier proyecto de unificación cultural y reafirma, además, la identificación de las personas con sus referentes culturales inmediatos. Y el panorama se completa, por último, con el ocaso de la ilusión del Estado culturalmente homogéneo (Parekh 1999). La idea subyacente es la siguiente: las sociedades se han caracterizado desde antiguo por su amplia diversidad y su pluralismo cultural. Antes, esa diversidad se aplastaba bajo el modelo del ciudadano 'normal' (hombre no discapacitado, propietario, heterosexual y blanco), y quien se desviara del modelo era excluido, marginado, silenciado o asimilado. Hoy, los grupos minoritarios demandan una nueva concepción de ciudadanía más inclusiva, que reconozca sus identidades y que dé cabida a sus diferencias (Cfr. Kymlicka 2002: 327 y 328).

Estamos insertos en un mundo social complejo que se resiste, porfiadamente, a ser reducido a un esquema conceptual o epistémico puramente monista. Somos testigos de un fenómeno cada vez más evidente y que ya no puede ocultarse bajo la ilusión del Estado-nación. Vivimos en medio de un escenario mundial en el que la globalización, las migraciones y la diversidad étnico-cultural ha alterado radicalmente la antigua homogeneidad -real o impuesta- de muchos Estados democráticos, especialmente en Europa, pero no sólo en ella (Torbisco 2001: 469). Chile, por supuesto, no escapa a esta realidad y se torna impostergable la reflexión y posterior construcción de teorías suficientemente sensibles ante esa evidencia, pero a la vez adecuadamente robustas para hacer frente al desafío apuntado sin que ello implique la fragmentación del Estado.

Tres son los grandes grupos de respuestas teóricas que podemos encontrar en la literatura (por ejemplo, Pérez de la Fuente 2004 y 2005) para hacer frente al desafío multicultural representado en la pregunta que he apuntado denantes: el liberalismo igualitario (V. gr. Barry 2002; Habermas 2003; Okin, 1999; y Rawls 2002), el culturalismo liberal (entre otros, Kymlicka 1996, 2003, 2007; Miller 2000; Raz, 2001; y Spinner1994) y el multiculturalismo o, mejor dicho, los multiculturalismos (en especial, Kukathas 1992a, 1992b, 1998; Parekh 2000 y 2006; y Young 2000). 
En primer lugar, el liberalismo igualitario -corregido después de la ofensiva comunitarista como ya se ha descrito- ha terminado por reconocer que el agente moral que escoge los principios de justicia no es anterior a sus fines y, por lo mismo, se encuentra vinculado y conectado a diferentes marcos cualitativos culturales que definen su identidad. Con todo, debido a la separación entre la esfera pública gobernada por la razón pública y la esfera privada donde los ciudadanos pueden relacionarse libremente con las concepciones del bien que sean consistentes con los límites impuestos desde la justificación pública, el liberalismo termina por ser ciego frente a las culturas privatizándolas. Así, por ejemplo, Habermas sostiene que "la integración ética de grupos y subculturas con sus propias identidades colectivas debe encontrarse, pues, desvinculada del nivel de la integración política, de carácter abstracto, que abarca a todos los ciudadanos en igual medida” (Habermas 1999: 215). La consecuencia normativa e institucional de esta aproximación es, siguiendo a Barry, evidente: los ciudadanos pueden, en ejercicio de su autonomía, llevar adelante los planes de vida que incorporen y mezclen, si lo desean, diversos referentes culturales. Postular que el liberalismo privatiza las formas de vida que no son liberales es equivalente a decir que el liberalismo «hace su trabajo», o sea, que los miembros de grupos no-liberales gozan, en condiciones de igualdad, de los mismos derechos que cualquier otro. Siempre que ellos lo elijan, pueden sentirse perfectamente libres de participar junto a otros en la observancia de una fe religiosa o adherir a una cultura que sea autocrática, misógina e intolerante. Pero los términos en los que ellos puedan hacer esto deben ser los mismos a los que se someten todos los ciudadanos. Lo anterior es la esencia de una sociedad gobernada por principios liberales e implica defender la tesis central de que no es función del Estado promover la diversidad cultural, puesto que ello podría poner en peligro la promoción de un marco que asegure elecciones autónomas, el auténtico deber del Estado (Cfr. Barry 2002: 123-125) ${ }^{1}$.

En segundo lugar, el culturalismo liberal se sitúa en una posición que busca destacar la relevancia moral de la identidad cultural protegiendo las diversas culturas nacionales y étnicas, pero garantizando la vigencia de principios liberales (Kymlicka 2003: 59). El punto de partida de esta posición es comprender la relevancia del contexto social como requisito para que exista una elección autónoma auténtica y significativa. Como sostiene Raz, "sólo a través de la socialización en una cultura podemos canalizar las opciones que dan sentido a la vida” (Raz 2001: 192). Esta postura se conecta con la tesis comunitarista que defiende la naturaleza contextual de los seres humanos, pero la sobrepasa porque la importancia de ésta tiene como objetivo asegurar a cada persona un marco de opciones cualitativamente significativas. De modo que las culturas ya no son relegadas a la esfera privada como plantea el liberalismo igualitario, sino que juegan un papel fundamental en la conformación de ciertos aspectos decisivos en la construcción de una identidad

\footnotetext{
${ }^{1}$ He revisado con detención la postura de Barry en Villavicencio (2010).
} 
nacional como es el caso de la lengua, la educación o los símbolos nacionales (Kymlicka 1996: 152-164).

Ahora bien, y este es el siguiente paso del culturalismo liberal, los Estados modernos están constituidos, habitualmente, por diversos grupos étnicos y/o nacionales lo que nos obliga a abandonar el falso discurso de la neutralidad y aceptar que al interior de esos Estados habrán de tomarse inevitables decisiones culturales entre los diferentes grupos nacionales y étnicos. Emerge así la necesidad de reconocer derechos de grupo, en función de una ciudadanía diferenciada y determinada culturalmente, para dotar a las minorías culturales de los mecanismos necesarios para enfrentar las discriminaciones que pueden padecer a manos de la cultura mayoritaria. Luego, para el culturalismo liberal, la dicotomía entre derechos colectivos y derechos individuales es errónea, puesto que deben distinguirse dos significados de derechos colectivos: por un parte, se encuentran los derechos externos, esto es, aquellos que reclaman la facultad del grupo -étnico o nacional- a ser tratado con igual consideración y respeto que otros grupos equivalentes (a modo de ilustración, los derechos a una lengua propia, el derecho a un estatuto especial de posesión de tierras o el derecho al gobierno autónomo o, al menos, a instituciones parcialmente autónomas); y, por otra, se encuentran los derechos internos, es decir, aquellos que reclaman el derecho del grupo de coaccionar a sus miembros impidiéndoles revisar su concepción del bien (por ejemplo, el derecho a sancionar a los individuos que se aparten de las creencias colectivas). Pues bien, los derechos colectivos externos son perfectamente coherentes con una teoría liberal de los derechos que pretende fomentar la autonomía y los derechos colectivos internos son inadmisibles (Kymlicka 1996: 57-76). Así, por ejemplo, es perfectamente válido en un Estado limitar el poder político y económico ejercido por la mayoría sobre un determinado grupo étnico con el objeto de asegurar que los recursos y las instituciones de que depende ese grupo minoritario no sean vulnerables a las decisiones de la mayoría. Por el contrario, es inválida cualquier acción del grupo étnico o cultural que tenga por objeto limitar la libertad de sus propios miembros en nombre de la solidaridad grupal o de pureza cultural.

En tercer lugar, el multiculturalismo adopta una posición crítica frente a ambas alternativas. Ahora bien, a diferencia del liberalismo igualitario y del culturalismo liberal, el multiculturalismo difícilmente puede caracterizarse como una tradición homogénea de pensamiento ya que carece, a diferencia del liberalismo, de fundadores o textos canónicos (Parekh 2002: 133150). Por esta razón, parece más adecuado hablar de multiculturalismos (De Lucas 2001: 61-102) que sólo tendrían en común la defensa de una estrategia multiculturalista, cuyo fin es sostener como tarea primordial del Estado la promoción de la diversidad cultural mediante una decidida política de la diferencia que supone redefinir los contornos de la justicia más allá del paradigma de la distribución para transitar al paradigma del reconocimiento (Young 2000: 31-69). La perspectiva multiculturalista considera: a) que los seres humanos existimos incardinados en las culturas en las que estamos insertos; b) que la visión de una cultura no puede ser sino 
limitada por lo que la diversidad cultural, además de ser inevitable, es enriquecedora al mostrar las posibilidades de la condición humana; y c) que las culturas se estructuran internamente de forma dinámica y plural (Parekh 2006: 336-344). Y si lo anterior es efectivo, la única opción viable es potenciar y permitir un diálogo entre las culturas que pueda llegar a ser provechoso para hacerse consciente de la propia particularidad y, además, para situarse ante la diferencia de forma positiva e inclusiva modificando, por ende, su carga estigmatizante (Cfr. Salas 2003).

He descrito, muy sucintamente, las tres grandes concepciones modelos que construyen una respuesta para hacerse cargo del fenómeno del multiculturalismo. Quisiera ahora justificar la pertinencia del que considero el único camino satisfactorio por recorrer, esto es, lograr articular una propuesta conciliatoria que permita equilibrar las pretensiones universalistas del liberalismo igualitario y las demandas particularistas y diferenciadas de las formas más radicales del multiculturalismo al interior de Estados multinacionales o pluriétnicos que no aceptarán la desintegración.

Por una parte, el liberalismo debe ser descartado pues la tesis de que es posible, para todo agente moral, deducir de una cierta concepción de la naturaleza humana un conjunto de principios morales universales, imparciales y neutrales se sustenta en endebles presupuestos metaéticos y conduce a conclusiones normativas espurias. Así pues, al menos es muy improbable defender, todavía, que el catálogo liberal de derechos individuales se sitúa neutral e imparcialmente frente a los diferentes modos de vida. Al contrario, ese catálogo supone elevar a ciertos modos de vida particulares al altar de la imparcialidad, transformando a la cultura dominante en la única adecuadamente justa que ofrecería a los modos de vida minoritarios la oportunidad de abandonar sus horizontes de sentido herederos, casi con seguridad, de la ignorancia o la barbarie. Tal como apunta Kymlicka, el problema del liberalismo es obviar que cuando los miembros de la cultura dominante se quejan de que no comprenden por qué los integrantes de la cultura minoritaria se comprometen tanto con el reconocimiento y la expresión de su propia cultura, ellos hacen precisamente lo mismo cuando les exigen que se integren cuanto antes a la sociedad mayoritaria, construida sobre la base de su propia identidad societal (Kymlicka 2004: 42). Para los grupos que se sitúan en los márgenes o fuera de la concepción tradicional de ciudadanía, lo fundamental es que se vinculan con sus fines y su propia identidad de manera no-electiva y, por lo mismo, no basta con que la organización política asegure un marco igualitario donde las personas escogen sus fines y vínculos, sino que debe dárseles algún grado de protección pues sus horizontes de sentido se encuentran amenazados, en todo momento, por la cultura mayoritaria.

Por otra parte, las formas más extremas de multiculturalismo también deberían ser desechadas pues no sólo defienden que los valores son internos a cada cultura, sino que además afirman la inconmensurabilidad radical entre culturas debido a una visión esencialista de las mismas que privilegia la estructura colectiva sobre los individuos que la componen. 
Esta postura sencillamente desdeñaría la posibilidad de un diálogo intercultural por ser epistémicamente imposible y políticamente inútil. Se transformaría, entonces, en una postura que clausura cualquier debate haciendo irrelevante toda aproximación a los problemas que nos plantea el desafío multicultural.

Como puede verse, ambas tesis_la del liberalismo ortodoxo y la del multilculturalismo radical- terminarían provocando, a pesar de sus visiones metaéticas y normativas tan disímiles, efectos relativamente similares: la creación o profundización de minorías nacionales y culturales excluyentes proclives, al menos potencialmente, al uso de medios violentos. El liberalismo alimentaría este poco alentador panorama invisibilizando las culturas minoritarias y privatizándolas al espacio anecdótico del folclore confiando en que pronto serán colonizadas una vez que sus miembros sean apropiadamente ilustrados. El multiculturalismo fuerte, por su parte, nutriría este escenario provocando un enfrentamiento entre la cultura dominante y las culturas minoritarias acarreando el efecto paradojal de que los grupos respecto de los cuales se arroga representación quedarían a merced de la cultura dominante, dándole a ésta última la legitimidad necesaria para presentar las demandas de los grupos minoritarios como balcanizantes sin más.

\section{Conclusiones}

Podría pensarse, a estas alturas, que esa vía intermedia que propongo habremos de transitar necesariamente sería, entonces, el culturalismo liberal de autores como Kymlicka y Raz. Pero esta es una conclusión apresurada. La tesis de estos pensadores liberales (sensibles eso sí -claro está- al problema de las minorías étnicas) se enfrenta a un problema de difícil solución como el propio filósofo canadiense reconoce. Aun cuando el enfoque liberal culturalista se ha posicionado rápidamente como la posición consensuada dentro de las filas del liberalismo corregido (algo que en todo caso Barry pone en duda enfáticamente, cfr. 2002: 6 y 7), éste presenta un grave problema: existen ciertos derechos de las minorías que, antes de sostener la autonomía individual, la socavan (grupos no liberales que restringen la libertad de todos o de algunos de sus miembros). Y ya sabemos que los derechos colectivos internos no son aceptados por esta corriente. Así pues, el culturalismo liberal termina por cuadrarse con Rawls cuando se pone en peligro el valor de la autonomía, exponiéndose en este punto a reproches similares y dejando sin respuesta la pregunta que anuncié antes: ¿por qué deberíamos tratarnos como semejantes si no nos vemos, desde nuestras culturas e identidades particulares, en ningún caso como semejantes?, o sea, ¿por qué habríamos de distribuir entre todos el valor de la autonomía -lo que justifica el rechazo de los derechos colectivos internos- si choca con la identidad cultural de la cual se nutre nuestra propia concepción del yo? 
Así pues, esa vía intermedia debería ser alguna forma de tesis multiculturalista débil como respuesta normativa-institucional al pluralismo cultural. Probablemente, y sólo conjeturo, esa propuesta conciliatoria, siguiendo a Parekh (2006: 336-344), debería reconocer, por un lado, la relevancia moral de la identidad cultural apoyándose en una adecuada interpretación del valor moral de las culturas que escape al mismo tiempo, por otro lado, de los tópicos que usualmente se asocian a las posiciones multiculturalistas. ¿Cómo lograrlo? Primero, afirmando que los seres humanos estamos culturalmente constituidos, pues nos desarrollamos y vivimos en un mundo culturalmente estructurado, organizando nuestras vidas y relaciones sociales en términos de un sistema de significados y valoraciones que le dan un valor considerable a nuestras identidades culturales, pero que no implica que estemos determinados por la cultura en la que nos insertamos por haberla heredado y aceptado acríticamente, convirtiéndonos en sujetos incapaces de evaluar sus creencias y prácticas, sino más bien como agentes capaces de revisarla reflexivamente o, en casos más excepcionales, abandonarla -total o parcialmente- comprendiendo y haciendo propia otras.

Segundo, reconociendo que las diferentes culturas representan diversos sistemas de significados y visiones de la vida buena, haciendo que la diversidad cultural sea no sólo inevitable sino deseable. Cada uno de nosotros realiza un limitado espectro de capacidades humanas y sólo percibe una parte de la totalidad de la existencia humana, necesitando otras visiones para entenderla mejor, expandiendo nuestro horizonte intelectual y moral, agudizando nuestra imaginación y guardándonos de la fascinación por absolutizar la propia cosmovisión. Partir, de buena fe, del supuesto que ninguna cultura carece completamente de valor; mereciendo al menos algo de respeto por lo que significa para sus miembros y la energía creativa que supuso y supone para ellos. Entender que ninguna cultura es perfecta, ni tiene el derecho de imponerse a otras, y que las culturas cambian mejor desde la crítica interna. Pero, junto con ello, y debido a que cada cultura es connaturalmente limitada, promover el diálogo entre ellas como algo mutuamente beneficioso. Diálogo que es posible, en fin, porque cada cultura acepta a las otras como participantes iguales en el plano discursivo, las que deben ser tomadas en cuenta como fuente de nuevas ideas.

Tercero, caracterizando a las culturas como internamente plurales aunque con pretensiones de coherencia interna, representando un juego dialógico entre diferentes tradiciones y estados de pensamiento. Las culturas surgen en una interacción con otras culturas y son, a la vez, permeables, sujetas a influencias externas que se interpretan y asimilan de una forma autónoma.

Por todo lo dicho, ahora de la mano de Pérez de la Fuente (2005: 121 y 122), ésta es una versión del multiculturalismo que patrocina el valor de la pertenencia cultural sin caer en el mecanicismo social ni en la caricatura esencialista y disgregadora con la que algunas posiciones caracterizan al multiculturalismo, pienso especialmente en los trabajos de Barry. Con todo, persiste lo que yo llamaría la paradoja multiculturalista, lo que hace 
que la gestión del pluralismo cultural sea siempre un tema de suyo complejo. Y para graficarla recurriré nuevamente al ejemplo de la violación de los derechos de las mujeres como grupo conceptualmente minoritario. Pensemos en las culturas tradicionales patriarcales que se sustentan en prácticas claramente discriminatorias contra las mujeres. Okin ha criticado, con razón, que la reivindicación de los derechos de grupo, que buscan preservar una cultura, pueden suponer vulneraciones graves e intolerables a la igualdad entre el hombre y la mujer (1998). Ésta es una perspectiva insoslayable que nos retrotrae a la cuestión de si el multiculturalismo es una opción progresista o conservadora. Lo que debemos determinar es si la cultura es una justificación válida para no aplicar un catálogo de derechos básicos y, en términos más abstractos, si existen razones persuasivas para no distribuir con pretensiones de universalidad el valor de la autonomía. Quienes defendamos una posición multiculturalista nos encontraríamos en medio del dilema de considerar como válidas aquellas culturas -desgraciadamente demasiadas desde mi propia cosmovisión- que suponen la discriminación de la mujer o, en cambio, sostener normativamente la igualdad de la mujer. Pero en este último caso, apelaríamos a valores de moralidad crítica universales que no todas las versiones del multiculturalismo podrían justificar.

No obstante, esa paradoja, creo, es sólo aparente. Si concedemos que una cultura puede justificar la no aplicación del catálogo de derechos a un grupo -en nuestro ejemplo las mujeres- estaríamos cayendo en la versión tópica y caricaturesca del multiculturalismo de la que, precisamente, las mismas tesis multiculturalistas pretenden zafarse. Si una cultura cae en este vicio estaría siendo inconsistente con la única versión plausible del multiculturalismo, pues encasillaría, petrificaría y esencializaría la cultura a costa de la autonomía de algunos de sus miembros impidiendo que éstos revisen y cuestionen su pertenencia a la cultura. La cultura dejaría de ser, como supone Parekh, permeable e influenciable externamente haciendo que todo cuestionamiento sobre el modo en que debemos enfrentar el desafío multicultural sea trivial. Una posición como la descrita debe ser apartada del debate porque clausura definitivamente -desde dentro- cualquier nivel de discusión, haciéndola inútil. Lo crucial aquí es que tanto liberales igualitarios, culturalistas liberales y multiculturalistas deberían estar de acuerdo. La cuestión clave es saber transitar del monismo excluyente de la diversidad, que considera a otras formas de vida inferiores fruto de la ignorancia y de la barbarie, al pluralismo de valores que considera que existen algunos valores universales pero diversas formas de vida. Ése es el trasfondo metaético correcto en el cual deben situarse las cuestiones relacionadas con el pluralismo cultural.

Aclarados los pilares sobre los cuales se edificaría esa posición multiculturalista débil, estamos en condiciones de enumerar, para terminar, los puntos centrales que resumen la postura que he intentado defender: (1) Una adecuada interpretación de la justicia no sólo tiene que ver con el paradigma de la distribución, sino con el paradigma del reconocimiento. (2) Comparto la tesis social comunitarista, diversa de la ontología individualista, que se traduce en la importancia del contexto en la conformación 
de la identidad humana y su consecuente protección. (3) Defiendo la imposibilidad de hallar un «punto de vista moral» totalmente imparcial y neutral y, por ende, reconozco la función ideológica del principio de neutralidad liberal, sustentado en una concepción del bien igualmente competitiva que otras. (4) Habrá de consolidarse una definición amplia de cultura que comprenda una definición de grupo no esencialista, sino contextual, dinámica y relacional. (5) Se debe desmitificar la absolutización de los principios liberales como criterios universalmente válidos que sirvan de parámetro exclusivo para valorar otras culturas. En su defecto, debe afirmarse la particularidad cultural de la democracia liberal reivindicando el papel de las diferentes culturas y la idoneidad de un diálogo intercultural que se tome en serio, para entrar en el debate, el igual valor de las culturas aunque tengamos buenas razones, al clausurar el debate, para considerar ciertas prácticas culturas inaceptables. Y (6) Valoro positivamente la diversidad cultural y considero que es, en consecuencia, una función del Estado impulsar una política de la diferencia, descartando el modelo de asimilación que supone la eliminación de las diferencias en la esfera pública, típico de los Estados insensibles al desafío multicultural. Al contrario, debemos considerar la diferencia social no como fuente de estigmatización, sino de forma emancipatoria como un mecanismo de lucha política para conseguir una democracia más inclusiva. Lo que exige, sin duda, la implementación de medidas que reconozcan ciertos derechos colectivos (Cfr. Pérez de la Fuente 2005: 105-107). 


\section{Bibliografía}

Barry, Brian (2002), Culture and Equality. An Egalitarian Critique of Multiculturalism. Harvard University Press, Cambridge.

Beltrán, Elena (2002), “Diversidad y deberes cívicos: liberalismo, ciudadanía y multiculturalismo”, en Estado, justicia, derechos, Elías Díaz y José Luis Colomer (eds.). Alianza, Madrid, pp. 371-407.

Comanducci, Paolo (1996), "La imposibilidad de un comunitarismo liberal”, en Tolerancia y minorías. Problemas jurídicos y políticos, Luis Prieto Sanchos (coord.). Ediciones de la Universidad de Castilla-La Mancha, Cuenca, pp. 13-26 (traducción de Marina Gascón).

de Lucas, Javier (2001), "La(s) sociedad(es) multicultural(es) y los conflictos políticos y jurídicos”, en La multiculturalidad. Consejo General del Poder Judicial, Madrid, pp. 61-102.

Dworkin, Ronald (1993), Ética privada e igualitarismo político. Paidós, Barcelona (traducción de Antoni Domènech).

Idem (1996a), La comunidad liberal. Siglo del Hombre Editores, Santafé de Bogotá (traducción de Claudia Montilla).

Idem (1996b), “Objectivity and Truth: You'd Better Believe It”, en Philosophy and Public Affairs, Volumen 25, N² 2, pp. 87-139.

Idem (2000), Sovereign Virtue. The Theory and Practice of Equality. Harvard University Press, Cambridge.

Facchi, Alessandra (1999), "Mutilaciones genitales femeninas y derecho positivo", en Derechos de las minorías en una sociedad multicultural, Javier de Lucas (ed.). Consejo General del Poder Judicial, Madrid, pp. 143-167.

Gargarella, Roberto (1999), Las teorías de la justicia después de Rawls. Paidós, Barcelona.

Habermas, Jürgen (1999), "La lucha por el reconocimiento en el Estado democrático de derecho”, en La inclusión del otro. Paidós, Barcelona, pp. 189-227 (traducción de Juan Carlos Velasco).

Idem (2003), “De la tolerancia religiosa a los derechos culturales”, en Claves de razón práctica, $\mathrm{N}^{\circ} 129$, pp. 4-10.

Kukathas, Chandran (1992a), “Are There Any Cultural Rights?”, en Political Theory, Volumen 20, $\mathrm{N}^{\circ}$ 1, 1992, pp. 105-139.

Idem (1992b), “Cultural Rights Again: A Rejoinder to Kymlicka”, en Political Theory, Volumen 20, $\mathrm{N}^{\circ} 4$, pp. 674-680. 
Idem (1998), "Liberalism and Multiculturalism: The Politics of Indifference”, en Political Theory, Volumen 26, № 5, pp. 686-699.

Kymlicka, Will (1996), Ciudadanía multicultural. Una teoría liberal de los derechos de las minorías. Paidós, Barcelona (traducción de Auleda Castells).

Idem (2002), Contemporary Political Philosophy. An Introduction, (2 ed.). Oxford University Press.

Idem (2003), La política vernácula. Nacionalismo, multiculturalismo y ciudadanía. Paidós, Barcelona (traducción de Tomás Fernández Aúz y Beatriz Eguibar).

Idem (2004), Estados, naciones y culturas. Almuzara, Córdoba (traducción y adaptación de Juan Jesús Mora).

Idem (2007), Multicultural Odysseys: Navigating the New International Politics of Diversity. Oxford University Press.

MacIntyre, Alasdair (1987), Tras la virtud. Crítica, Barcelona (traducción de Amelia Valcárcel).

Idem (1992), Tres versiones rivales de la ética. Enciclopedia, genealogía y tradición. Ediciones Rialp, Madrid (traducción de Rogelio Rovira).

Idem (1994), Justicia y racionalidad. Conceptos y contextos. Ediciones Internacionales Universitarias, Barcelona (traducción y presentación de Alejo José G. Sison).

Idem (2006), Ethics and Politics: Selected Essays. Cambridge University Press.

Miller, David (2000), Citizenship and National Identity. Polity Press, Cambridge.

Mulhall, Stephen y Swift, Adam (1996), El individuo frente a la comunidad. El debate entre liberales y comunitaristas. Ediciones Temas de Hoy, Madrid (traducción y presentación de Enrique López Castellón).

Nino, Carlos (1996), “Kant versus Hegel, otra vez”, en Política. Revista de Estudios sobre el Estado y la Sociedad, N 1, Paidós, Barcelona, pp. 123135.

Okin, Susan (1998), “Feminism and Multiculturalism: Some Tensions”, Ethics, July 1998, Volumen 108, º 4, pp. 661-684.

Idem (1999), Is multiculturalism bad for women? Princeton University Press. 
Parekh, Bhikhu (1999), "Political Theory and the Multicultural Society", en Radical Philosophy, Volumen 95. Obtenido el 10 de septiembre de 2009 en: http://www.radicalphilosophy.com/default.asp?channel_id= 2188\&editorial_id=10166

Idem (2002), "Barry and the dangers of liberalism”, en Multiculturalism Reconsidered. Culture and Equality and its Critics, Paul Kelly (ed.). Polity Press, Cambridge, pp. 133-150.

Idem (2006), Rethinking Multiculturalism. Cultural Diversity and Political Theory, (2 ed.). Palgrave Macmillan.

Peña, Carlos (2002), "Equilibrio reflexivo, constructivismo y razón pública. El problema de la realidad y la justificación en filosofía política”, en John Rawls: Estudios en su memoria, Revista de Ciencias Sociales, Edeval, Universidad de Valparaíso, N 47, pp. 333-437.

Pérez de la Fuente, Oscar (2004), La polémica liberal comunitarista. Paisajes después de la batalla. Dykinson, Madrid.

Idem (2005), Pluralismo cultural y derechos de las minorías. Dykinson, Madrid.

Idem (1971), A Theory of Justice. Oxford University Press, Londres (hay disponible una traducción de María Dolores González, Teoría de la justicia, Fondo de Cultura Económica, Buenos Aires, 1979).

Idem (1986), Justicia como equidad. Materiales para una teoría de la justicia. Tecnos, Madrid (traducción de Miguel Ángel Rodilla).

Idem (1996), El liberalismo político. Crítica, Barcelona (traducción de Antoni Domènech).

Idem (2001a), “Una revisión de la idea de razón pública” en El derecho de gentes. Paidós, Barcelona, pp. 155-205 (traducción de Hernando Valencia Villa).

Idem (2001b), Lecciones sobre la historia de la filosofía moral, compilado por Barbara Herman. Paidós, Barcelona (traducción de Andrés de Francisco).

Idem (2002), La Justicia como equidad. Una reformulación. Paidós, Barcelona (traducción de Andrés de Francisco).

Raz, Joseph (1986), The Morality of Freedom. Oxford University Press. Idem (2001), La ética en el ámbito de lo público. Gedisa, Barcelona (traducción de María Luz Melon).

Rorty, Richard (1991), Contingencia, ironía y solidaridad. Paidós, Barcelona (traducción de Alfredo Eduardo Sinnot, revisión de Jorge Vigil). 
Idem (1996), Objetividad, relativismo y verdad. Escritos filosóficos 1. Paidós, Barcelona (traducción de Jorge Vigil Rubio).

Salas Astraín, Ricardo (2003), Ética intercultural. (Re)Lecturas del pensamiento latinoamericano. Ediciones UCSH, Santiago de Chile.

Sandel, Michael (1996), Democracy's Discontent. America in Search of a Public Philosophy. Harrvard University Press, Cambridge.

Idem (1998), Liberalism and the Limits of Justice, (2 ed.). Cambridge University Press, Nueva York.

Idem (2000), El liberalismo y los límites de la justicia. Gedisa, Barcelona (traducción de María Luz Melon).

Idem (2006), Public Philosophy: Essays on Morality in Politics. Harvard University Press.

Seleme, Hugo O. (2004), Neutralidad y justicia. En torno al liberalismo político de John Rawls. Marcial Pons, Madrid.

Spinner, Jeff (1994), The Boundaries of Citizenship: Race, Ethnicity and Nationality in the Liberal State. Johns Hopkins University Press, Baltimore.

Taylor, Charles (1990), “Atomismo”, en Derecho y moral, Betegón, Jerónimo y de Páramo, Juan Ramón (dirección y coordinación). Ariel, Barcelona, pp. 107-124 (traducción de Silvia Mendlewicz y Albert Calsamiglia).

Idem (1994), La ética de la autenticidad. Paidós, Barcelona (traducción de Pablo Carbajosa Pérez).

Idem (1996a), Fuentes del yo. La construcción de la identidad moderna. Paidós, Barcelona (traducción de Ana Lizón y revisión técnica de Ramón Alfonso Díez).

Idem (1996b), “La diversidad de bienes”, en Política. Revista de Estudios sobre el Estado y la Sociedad, N 1, Paidós, Barcelona, pp. 65-80 (traducción de Sebastián Abad).

Idem (1997), Argumentos filosóficos. Ensayos sobre el conocimiento, el lenguaje y la modernidad. Paidós, Barcelona (traducción de Fina Birulés Bertrán).

Torbisco, Casals, Neus (2001), Minorías culturales y derechos colectivos: Un enfoque liberal (tesis doctoral). Universitat Pompeu Fabra, Barcelona. Obtenido el 3 de septiembre de 2009 de: www.tdx.cesca.es/TESIS_UPF/ AVAILABLE/TDX-1212101-123858//tntc1de1.pdf

Villavicencio Miranda, Luis (2007), “La concepción política de la perso- 
na y las visiones constitutivas del bien”, en Ideas y Valores. Revista Colombiana de Filosofía, Universidad Nacional de Colombia, $\mathrm{N}^{\circ} 135$, diciembre de 2007, pp. 29-49.

Idem (2009), “Algunas críticas a la idea de razón pública rawlsiana”, en Revista de Derecho de la Pontificia Universidad Católica de Valparaíso, 2009, volumen 32, $\mathrm{N}^{\circ} 1$, pp. 533-557.

Idem (2010), "Privatizando la diferencia: El liberalismo igualitario y el pluralismo cultural”, en Revista de Derecho (Valdivia), julio 2010, volumen XXIII, $N^{\circ} 1$, en prensa.

Walzer, Michael (1993), Las esferas de la justicia. Una defensa del pluralismo y la igualdad. Fondo de Cultura Económica, México D.F. (traducción de Heriberto Rubio).

Idem (1996), "La crítica comunitarista del liberalismo”, en Política. Revista de Estudios sobre el Estado y la Sociedad, № 1, Paidós, Barcelona, pp. 47-64 (traducción de Sebastián Abad).

Idem (1998), Tratado sobre la tolerancia. Paidós, Barcelona (traducción de Francisco Álvarez).

Idem (2006), Politics and Passion: Toward a More Egalitarian Liberalism. Yale University Press.

Young, Iris Marion (2000), La justicia y la política de la diferencia. Cátedra, Madrid (traducción de Silvina Álvarez). 